\title{
Functional Characteristics of Two Human MATE Transporters: Kinetics of Cimetidine Transport and Profiles of Inhibition by Various Compounds
}

\author{
Kin-ya Ohta ${ }^{1}$, Katsuhisa Inoue ${ }^{1}$, Tomoya Yasujima ${ }^{1}$, Munenori Ishimaru ${ }^{2}$, Hiroaki Yuasa ${ }^{1}$ \\ ${ }^{1}$ Graduate School of Pharmaceutical Sciences, Nagoya City University, Nagoya 467-8603, Japan \\ ${ }^{2}$ School of Pharmacy, Aichi Gakuin University, Nagoya 464-8651, Japan
}

Received, September 14, 2009; Revised, November 11, 2009; Accepted, December 8, 2009; Published, December 26, 2009.

\begin{abstract}
Purpose. Human multidrug and toxin extrusion protein 1 (hMATE1) and hMATE2-K are organic cation $/ \mathrm{H}^{+}$ antiporters that have recently been identified and suggested to be involved in the renal brush border secretion of various organic cations. Information about functional characteristics of them has been accumulating, but still insufficient to fully understand their functions and respective roles. The present study was conducted to help clarify them. Methods. The cDNAs of hMATE1 and hMATE2-K were isolated from human kidney total RNA by RT-PCR. HEK293 cells were stably transfected with hMATE1 and hMATE2-K, and the cellular uptakes of $\left[{ }^{3} \mathrm{H}\right]$ cimetidine and $\left[{ }^{14} \mathrm{C}\right]$ tetraethylammonium (TEA) were evaluated. Results. It was first found that both hMATE1 and hMATE2-K can transport cimetidine with high affinities, indicated by small Michaelis constants of $8.00 \mu \mathrm{M}$ and $18.18 \mu \mathrm{M}$, respectively. These were much smaller than those for TEA (366 $\mu \mathrm{M}$ and $375 \mu \mathrm{M}$, respectively, for hMATE1 and hMATE2-K). Subsequent investigation using cimetidine as a probe substrate into the profiles of inhibition of the two hMATEs by various compounds indicated that they are similar in principle but different to some extent in substrate recognition, reflecting the modest differences in amino acid sequences between them. In fact, cimetidine transport by hMATE1 was correlated to that by hMATE2-K, which is $65 \%$ similar to hMATE1, but not as good as to that by rat MATE1, which is $86 \%$ similar. Conclusions. Cimetidine was demonstrated to be a high affinity substrate of both hMATEs. Subsequent evaluation of the inhibition of hMATEs by various compounds indicated no major difference in function or role between hMATE1 and hMATE2-K.
\end{abstract}

\section{INTRODUCTION}

The organic cation transport system in the renal tubules has long been known to be involved in the urinary excretion of xenobiotics and endogenous metabolites with cationic nature, and recent studies have unveiled its molecular entities at the basolateral and brush border membranes. Multidrug and toxin extrusion protein 1 (MATE1) and MATE2-K are those which have most recently been identified and demonstrated to mediate the transport of organic cations by a $\mathrm{H}^{+}$-coupled antiport mechanism at the brush border membrane $(1,2)$. hMATE2-K is a splice variant of hMATE2, which was identified together with hMATE1 in the first study on mammalian MATEs by Otsuka et al (1). It has been suggested that hMATE2-K is specifically and abundantly expressed in the kidney, whereas hMATE2 and hMATE2-B, another splice variant of hMATE2, are expressed only poorly throughout the body (2). Therefore, hMATE2-K is presumably the major one among hMATE2 variants. It should be noted that, interestingly, the ortholog of hMATE2 is genetically deficient in rat and mouse, although MATE1 has been cloned in rat $(3,4)$ and mouse (5) as well as in human. MATE2-K may be needed in human, but not in rodents, for a role supplementary to or different from that of MATE1. However, the specific role of MATE2-K is uncertain yet.

Cimetidine is a typical substrate of organic cation transporters, including MATEs. This drug was shown to be recognized by rat MATE1 (rMATE1) with a high affinity indicated by $K_{\mathrm{m}}$ of $3.01 \mu \mathrm{M}$ (4). More recently, cimetidine was shown to inhibit hMATE1-mediated transport of fexofenadine by about $50 \%$ at its concentration of 3 - $30 \mu \mathrm{M}$ (6), suggesting its high affinity for hMATE1 with $\mathrm{IC}_{50}$ within that concentration range or below.

Corresponding Author: Katsuhisa Inoue, Ph.D., Department of Biopharmaceutics, Nagoya City University, Nagoya, Japan; E-mail address: kinoue@phar.nagoya-cu.ac.jp 
However, a much lower affinity of hMATE1 for cimetidine has also been suggested by an order of magnitude greater $K_{\mathrm{m}}$ of $170 \mu \mathrm{M}$ in another study (7). Thus, the affinity of hMATE1 for this important probe drug remains uncertain.

Thus, information about functional characteristics of the two hMATEs, hMATE1 and hMATE2-K, has been accumulating, but still insufficient to fully understand their functions and respective roles. We here report our attempts to further clarify the characteristics of them, focusing on the transport of cimetidine, in comparison with that of tetraethylammonium (TEA) as a probe substrate with a low affinity, and the profiles of inhibition by various exogenous and endogenous compounds. Characteristics of the hMATEs were also compared with those of rMATE1.

\section{MATERIALS AND METHODS}

\section{Materials}

$\left[{ }^{14} \mathrm{C}\right]$ TEA bromide $(2.4 \mathrm{mCi} / \mathrm{mmol})$ and $\left[{ }^{3} \mathrm{H}\right]$ cimetidine $(25.0 \mathrm{Ci} / \mathrm{mmol})$ were obtained from GE Healthcare Biosciences Co. (Piscataway, NJ, U.S.A.). TEA, cimetidine and geneticin were obtained from Sigma-Aldrich Co. (St. Louis, MO, U.S.A.), and restriction enzymes were from Toyobo, Co., Ltd. (Tokyo, Japan). All other reagents were of analytical grade and commercially obtained.

\section{Cell culture and stable transfection}

HEK293 cells were maintained at $37^{\circ} \mathrm{C}$ and $5 \%$ $\mathrm{CO}_{2}$ in Dulbecco's modified Eagle's medium (DMEM) with $10 \%$ fetal bovine serum (FBS), 100 units $/ \mathrm{ml}$ penicillin and $100 \mu \mathrm{g} / \mathrm{ml}$ streptomycin. The cDNAs of hMATE1 and hMATE2-K were cloned from the human kidney total RNA (Clontech, Mountain View, CA, U.S.A.) by reverse transcription (RT) and subsequent polymerase chain reaction (PCR) and introduced into a mammalian expression vector, pCI-neo (Promega, Madison, WI), as described previously (6), to prepare hMATE1/pCI-neo and hMATE2-K/pCI-neo plasmids. HEK293 cells were transfected with the hMATE1/pCI-neo, hMATE2-K/pCI-neo or pCI-neo, which was for mock cells, by the calcium phosphate coprecipitation method (8) and cultured in DMEM containing $10 \%$ FBS and $800 \mu \mathrm{g} / \mathrm{ml}$ geneticin for 2 to 3 weeks. Antibiotic-resistant clones were selected and tested for the transport of cimetidine as a probe substrate.

\section{Transport study in HEK293 cells}

HEK293 cells stably expressing hMATE1, those expressing hMATE2-K or mock cells $\left(1.5 \times 10^{5}\right.$ cells/well initially) were grown on 24-well plates coated with poly-L-lysine for $72 \mathrm{~h}$. To assess the functions of the hMATEs, which operate as organic cation $/ \mathrm{H}^{+}$antiporters, by the uptake of substrates into the cells, we used a method to generate an outward $\mathrm{H}^{+}$gradient by intracellular acidification (9). Before transport assays, cells in each well were washed with substrate-free uptake buffer $\left(130 \mathrm{mM} \mathrm{KCl}, 2 \mathrm{mM} \mathrm{KH} \mathrm{PO}_{4}, 1.2 \mathrm{mM}\right.$ $\mathrm{MgSO}_{4}, 1.0 \mathrm{mM} \mathrm{CaCl} 2,5 \mathrm{mM}$ glucose, and 20 $\mathrm{mM}$ HEPES, $\mathrm{pH}$ 7.4), incubated in the substrate-free uptake buffer supplemented with 20 $\mathrm{mM} \mathrm{NH} 4 \mathrm{Cl}(1.5 \mathrm{ml})$ for $10 \mathrm{~min}$, and thereafter incubated in the uptake buffer free of substrate and $\mathrm{NH}_{4} \mathrm{Cl}(1.5 \mathrm{ml})$ for $5 \mathrm{~min}$. Transport assays were started by replacing the substrate-free uptake buffer with uptake buffer containing a radiolabeled substrate $(0.25 \mathrm{ml})$. All the procedures were conducted at $37^{\circ} \mathrm{C}$. Assays were stopped by addition of ice-cold substrate-free uptake buffer $(1 \mathrm{ml})$, and the cells were washed two times with $2 \mathrm{ml}$ of the same buffer. The cells were solubilized in $0.5 \mathrm{ml}$ of $0.2 \mathrm{M} \mathrm{NaOH}$ solution containing $0.5 \%$ sodium dodecyl sulfate (SDS) at room temperature for $1 \mathrm{~h}$, and the associated radioactivity was measured by liquid scintillation counting, using $5 \mathrm{ml}$ of Clear-sol I (Nakarai Tesque, Inc., Kyoto, Japan) as a scintillation fluid, for the evaluation of uptake. Cellular protein content was determined by the method of Lowry et al. (10), using bovine serum albumin as the standard. The specific uptake by the introduced transporter, hMATE1 or hMATE2-K, was estimated by subtracting the uptake in mock cells from that in the transporter-transfected cells.

\section{Data analysis}

The saturable transport of each substrate, TEA and cimetidine, was analyzed by assuming Michaelis-Menten type carrier-mediated transport represented by the following equation: $v=V_{\max } \times$ $s /\left(K_{\mathrm{m}}+s\right)$. The maximum transport rate $\left(V_{\max }\right)$ 
and the Michaelis constant $\left(K_{\mathrm{m}}\right)$ were estimated by fitting this equation to the experimental profile of the uptake rate $(v)$ versus the substrate concentration (s), using a nonlinear least-squares regression analysis program, WinNonlin (Pharsight, Mountain View, CA, USA), and the reciprocal of variance as the weight. The $v$ in the presence of an inhibitor can be expressed as follows: $v=v_{0} /\left(1+\left(i / I C_{50}\right)^{n}\right)$. The half-inhibition concentration $\left(\mathrm{IC}_{50}\right)$ was estimated together with $v$ in the absence of inhibitors $\left(v_{0}\right)$ and the Hill coefficient ( $n$ ) by fitting this equation to the experimental profile of $v$ versus the inhibitor concentration (i). The parameters are presented as the computer-fitted ones with S.E.

Experimental data are represented as the means \pm S.E., and statistical analysis was performed by using analysis of variance (ANOVA) followed by Dunnett's test, with $\mathrm{p}<$ 0.05 considered significant.

\section{RESULTS}

\section{Kinetic characteristics of transport by hMATEs}

Throughout the present study, an outwardly directed proton gradient was generated by intracellular acidification by means of pretreatment with $\mathrm{NH}_{4} \mathrm{Cl}$, and the transport of cimetidine or TEA was assessed as an influx in exchange with proton. As shown in Figure $1 \mathrm{~A}$, the uptake of cimetidine $(50 \mathrm{nM})$ was much faster in HEK293 cells stably expressing hMATE1 and those expressing hMATE2-K than in mock cells transfected with empty pCI-neo vector, indicating its efficient uptake mediated by these hMATEs. Cimetidine uptake increased in proportion to time at least up to $30 \mathrm{~s}$ in all types of cells. Similar results were obtained for TEA $(10 \mu \mathrm{M})$, as shown in Figure 1B. Based on these results, an incubation period of $20 \mathrm{~s}$ was chosen in subsequent experiments to evaluate transport across the cellular membrane within the initial uptake phase.

The uptakes of cimetidine mediated by hMATE1 and hMATE2-K and those of TEA were all saturable and conformed to Michaelis-Menten kinetics (Figure 2 and Table 1).

For cimetidine, the $K_{\mathrm{m}}$ of hMATE2-K (18.18 $\mu \mathrm{M})$ was about 2-fold greater than that of hMATE1 $(8.00 \mu \mathrm{M})$, indicating that hMATE1 has a higher affinity than hMATE2-K. The $K_{\mathrm{m}}$ of rMATE1 (3.01 $\mu \mathrm{M})$ estimated in our earlier study (4) was closer to that of hMATE1. For TEA, the $K_{\mathrm{m}}$ of hMATE2-K
$(375 \mu \mathrm{M})$ was close to that of hMATE1 $(366 \mu \mathrm{M})$, indicating almost identical affinities. These $K_{\mathrm{m}}$ values were also comparable with that of rMATE1 $(260 \mu \mathrm{M})(4)$.

\section{A}

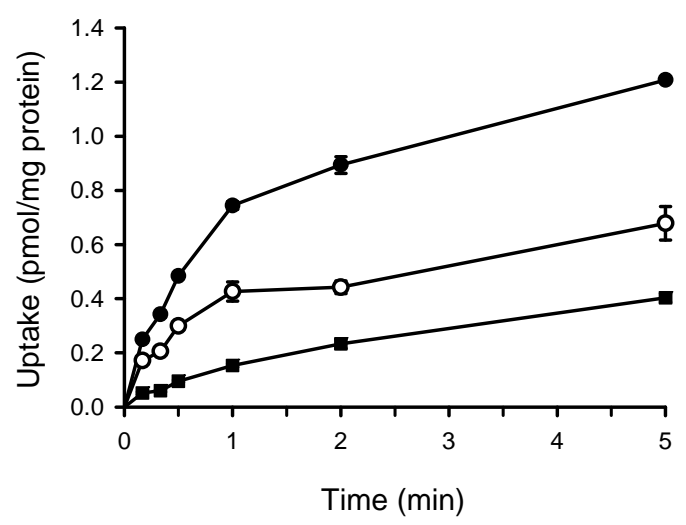

B

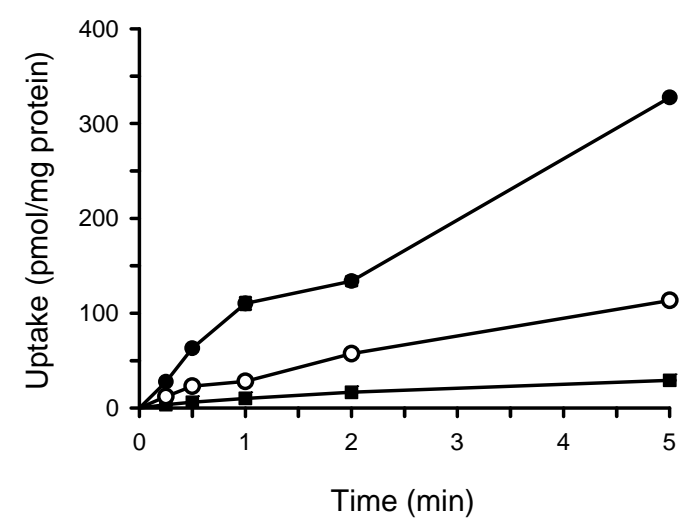

Figure 1. Time courses of the uptakes of cimetidine (A) and TEA (B) in HEK293 cells stably expressing hMATEs. The uptakes of $\left[{ }^{3} \mathrm{H}\right]$ cimetidine $(50 \mathrm{nM})$ and $\left[{ }^{14} \mathrm{C}\right] \mathrm{TEA}(10$ $\mu \mathrm{M})$ were evaluated at $37^{\circ} \mathrm{C}$ and $\mathrm{pH} 7.4$ after intracellular acidification by means of pretreatment with $\mathrm{NH}_{4} \mathrm{Cl}$ in HEK293 cells expressing hMATE1 (•), those expressing hMATE2-K (०) and mock cells ( $(\bullet)$. Data are represented as the means \pm S.E. $(n=3)$.

\section{Inhibition of hMATEs by various compounds}

To examine if hMATE2-K might have a different role than hMATE1 in renal secretion, we compared hMATE1 and hMATE2-K by assessing the effect of various exogenous and endogenous compounds on the specific uptake of cimtidine $(50 \mathrm{nM})$ by each of them (Table 2). 
A

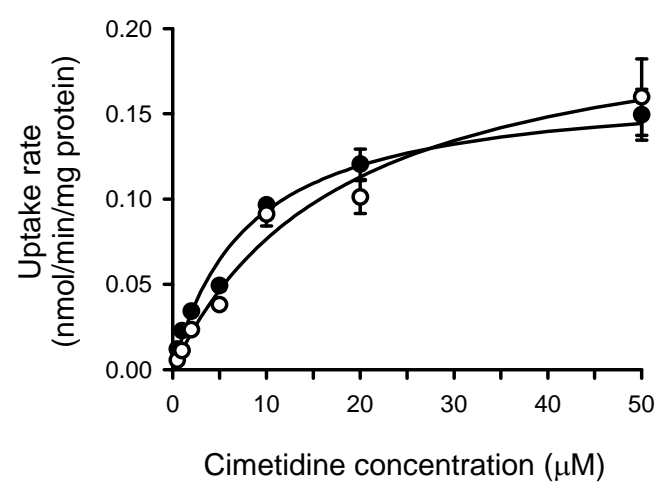

B

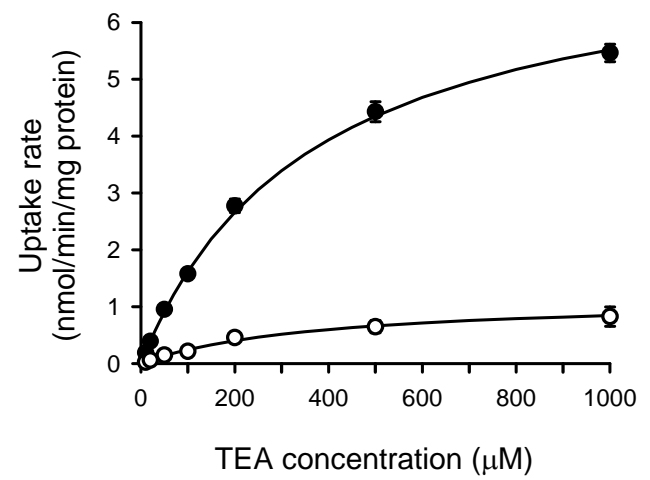

Figure 2. Concentration dependence of the uptakes of cimetidine (A) and TEA (B) mediated by hMATEs stably expressed in HEK293 cells. The uptakes of $\left[{ }^{3} \mathrm{H}\right]$ cimetidine $(50 \mathrm{nM})$ and $\left[{ }^{14} \mathrm{C}\right]$ TEA $(10 \mu \mathrm{M})$ were evaluated at $37^{\circ} \mathrm{C}$ and $\mathrm{pH} 7.4$ for $20 \mathrm{~s}$ after intracellular acidification by means of pretreatment with $\mathrm{NH}_{4} \mathrm{Cl}$ in HEK293 cells expressing hMATE1, those expressing hMATE2-K and mock cells. Data show the specific uptake rates by hMATE1 $(\bullet)$ and hMATE2-K $(\circ)$. Solid lines represent the computer-fitted profiles and the kinetic parameters are shown in Table 1. Data are represented as the means \pm S.E. $(n=3)$.
While many organic cations inhibited both hMATEs significantly, guanidine, histamine, nicotinamide and $\mathrm{N}^{1}$-methylnicotinamide (NMN) did neither of them or did only marginally. Corticosterone inhibited both hMATEs potently, although it is a neutral compound. p-Aminohippurate (PAH), an anionic compound, inhibited neither hMATE, as expected. Thus, these homologous transporters with a high similarity of $65 \%$ are similar in principle in profile of inhibition by various compounds.

It is notable, however, that estrone-3-sulfate, another anionic compound, was found to inhibit only hMATE1, indicating some difference between the two hMATEs. This compound was previously shown to also inhibit rMATE1 (4). It is also notable that diltiazem inhibited hMATE1 to an extent that is by about $80 \%$ and comparable with that for rMATE1, whereas it inhibited hMATE2-K to a lesser extent by about $30 \%$. Reflecting these, there is a trend that the extents of hMATE1 inhibition by various compounds are more comparable with those of rMATE1 inhibition than with those of hMATE2-K inhibition. Such a trend is more evident from the assessment of correlations in the uptakes of cimetidine by these transporters in the presence of various compounds. As shown in Figure 3, hMATE1-mediated uptake was better correlated with rMATE1-mediated uptake $\left(\mathrm{r}^{2}=0.96\right)$ than hMATE2-K-mediated uptake $\left(\mathrm{r}^{2}=0.85\right.$ and 0.89 , respectively, when data for compounds not tested for rMATE1 were excluded and included), with the regression line close to the line of identity in every correlation.

Table 1. Kinetic parameters of the uptakes of cimetidine and TEA mediated by hMATEs and rMATE1 stably expressed in HEK293 cells

\begin{tabular}{cccc}
\hline Compound & Transporter & $K_{\mathrm{m}}(\mu \mathrm{M})$ & $V_{\max }(\mathrm{nmol} / \mathrm{min} / \mathrm{mg}$ protein $)$ \\
\hline Cimetidine & hMATE1 & $8.00 \pm 0.26$ & $0.167 \pm 0.020$ \\
& hMATE2-K $^{*}$ & $18.18 \pm 0.69$ & $0.216 \pm 0.018$ \\
TEA & rMATE1 $^{a}$ & $3.01 \pm 0.21$ & $0.088 \pm 0.004$ \\
& hMATE1 & $366 \pm 17$ & $7.54 \pm 0.21$ \\
& hMATE2-K $^{a}$ & $375 \pm 68$ & $1.16 \pm 0.14$ \\
& rMATE1 $^{a}$ & $260 \pm 10$ & $5.41 \pm 0.21$ \\
\hline
\end{tabular}

Data are represented as the parameters \pm S.E. $(n=7)$ estimated by computer-fitting analyses of the data shown in Figure 2. ${ }^{a}$ Data for rMATE1 were cited from our previous report (4). $K_{\mathrm{m}}$, Michaelis constant; $V_{\max }$, maximum transport rate; TEA, tetraethylammonium. 
Among various cationic inhibitors, 4',6-diamino-2-phenylindole (DAPI), propidium iodide (PI) and ethidium bromide (EB), which are fluorescent compounds, would be of particular interest. They were all found to be recognized by both hMATEs with high affinities, as indicated by $\mathrm{IC}_{50}$ values smaller than about $30 \mu \mathrm{M}$ (Figure 4 and Table 3).

\section{DISCUSSION}

In the present study, we first characterized kinetically the hMATE1-mediated transport and hMATE2-K-mediated transport of cimetidine, in comparison with those of TEA. Kinetic analyses indicated that, for cimetidine, the $K_{\mathrm{m}}$ values of hMATE1 and hMATE2-K were $8.00 \mu \mathrm{M}$ and 18.18 $\mu \mathrm{M}$, respectively. For TEA, the $K_{\mathrm{m}}$ values of the former and the latter were $366 \mu \mathrm{M}$ and $375 \mu \mathrm{M}$, respectively. Thus, both hMATEs were suggested to have a much higher affinity for cimetidine than for TEA. The high affinity of hMATE1 for cimetidine is consistent with our earlier finding that the $\mathrm{IC}_{50}$ of cimetidine for hMATE1-mediated fexofenadine transport would not be greater than 3 to $30 \mu \mathrm{M}(6)$. Therefore, it is more likely that cimetidine is a good substrate of hMATE1 with the $K_{\mathrm{m}}$ of about $10 \mu \mathrm{M}$, although an order of magnitude greater $K_{\mathrm{m}}(170$ $\mu \mathrm{M})$ was previously reported (7). Cimetidine is also a good substrate of hMATE2-K, although its affinity for hMATE2-K was suggested to be a little lower than that for hMATE1. Those $K_{m}$ values observed for cimetidine in the present study are smaller than the smallest ones reported to date, which are $70 \mu \mathrm{M}$ (hMATE1) and $60 \mu \mathrm{M}$ (hMATE2-K) for topotecan. Cimetidine can be, hence, used as a high affinity probe substrate of both hMATEs, as well as of rMATE1.

In the presence of various inhibitors, hMATE1-mediated uptake was better correlated with rMATE1-mediated uptake than hMATE2-K-mediated uptake (Figure 3). It seems to be reasonable to find this because hMATE1 is more analogous to rMATE1 ( $86 \%$ similarity) than hMATE2-K (65\% similarity). These results indicate that there are some differences in recognition of some compounds between the two hMATEs, although the differences do not seem to be very profound, in accordance with the modest differences in amino acid sequences between them.
Tanihara et al. examined the substrate specificities of hMATE1 and hMATE2-K by evaluating transport of various compounds (7). They found that many compounds are transported by both hMATEs and also those for which the $K_{\mathrm{m}}$ values were determined are recognized with relatively close affinities, with differences in $K_{\mathrm{m}}$ by factors of not more than about 3, by the two hMATEs.

Differences in $\mathrm{IC}_{50}$ by a factor of, likewise, not more than about 3 were also found for a few fluoroquinolones and cephalosporines in the same study. Although cisplatin (11) and cephalexin (7) have been reported to inhibit only hMATE1, their $\mathrm{IC}_{50}$ values are as high as about $1 \mathrm{mM}$ and $6.5 \mathrm{mM}$, respectively, indicating that they are recognized only poorly. Thus, any major difference in function or role between the two hMATEs has not been demonstrated so far. The results in the present study are in line with those in such earlier studies and indicate that the role of hMATE2-K may not be beyond the one supplementary to hMATE1.

Interestingly, DAPI, PI and EB, which are fluorescent compounds, were all found to be recognized by both hMATEs with high affinities (Figure 4 and Table 3). They may be utilized as probe substrates for rapid assays of the functionality of the hMATEs, taking advantage of their fluorescent nature.

It is also notable that thiamine, a water soluble vitamin, was found to be a potent inhibitor of both hMATEs. However, although it has been reported that both hMATEs can transport thiamine, their transport activities for this vitamin are only modest (7). Therefore, the potent inhibitory activity of thiamine may not be very relevant to its transport.

In conclusion, cimetidine was demonstrated to be a high affinity substrate of both hMATEs, hMATE1 and hMATE2-K. Using cimetidine as a probe substrate, the profiles of inhibition of the two hMATEs by various compounds were examined, but there was suggested no major difference in function or role between them. Finally, the finding that DAPI, PI and EB, which are fluorescent compounds, are recognized by both hMATEs with high affinities would be of interest, as they may be utilized as probe substrates for rapid assays of the functionality of the hMATEs. 
A

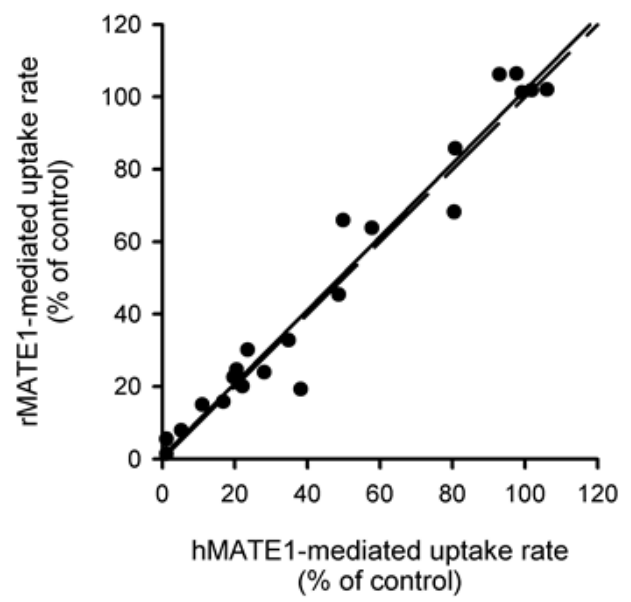

B

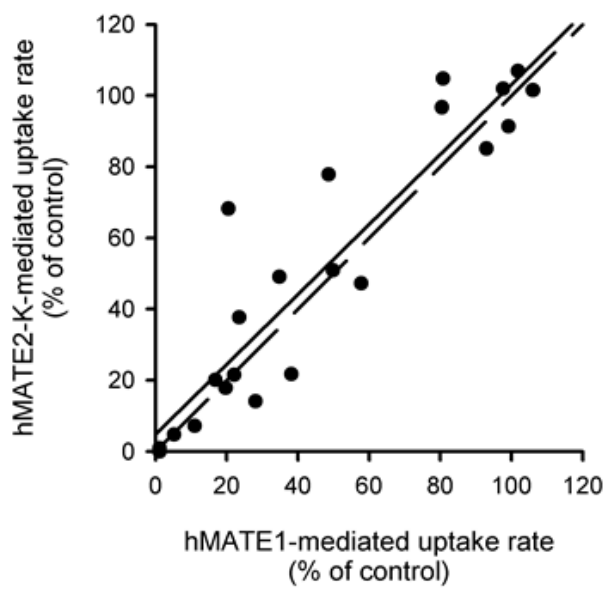

C

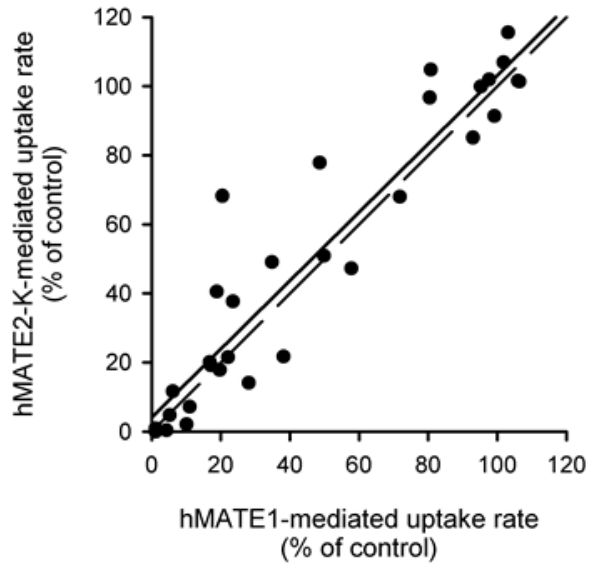

Figure 3. Correlations of cimetidine uptake by hMATE1 with those by rMATE1 and by hMATE2-K. Data shown in Table 2 were examined for correlations. Panel A is for rMATE1, and panels B and C are for hMATE2-K, where data for compounds not tested for rMATE1 were excluded in the former and included in the latter. Regression equations, which were estimated using the linear least-squares method, are as follows: $\mathrm{y}=1.01 \mathrm{x}+$ 0.78 with $\mathrm{r}^{2}=0.96, \mathrm{y}=0.98 \mathrm{x}+4.85$ with $\mathrm{r}^{2}=0.85$ and $\mathrm{y}$ $=0.99 \mathrm{x}+4.44$ with $\mathrm{r}^{2}=0.87$, respectively, in panels $\mathrm{A}, \mathrm{B}$ and $\mathrm{C}$, where $\mathrm{y}$ represents cimetidine uptake rate by rMATE1 or hMATE2-K and $\mathrm{x}$ represents that by hMATE1. Solid lines represent regression lines and broken lines represent the lines of identity (1:1) for reference.

Table 2. Effect of various compounds on cimetidine uptake mediated by hMATEs and rMATE1 stably expressed in HEK293 cells

\begin{tabular}{|c|c|c|c|c|c|}
\hline \multirow{2}{*}{ Category } & \multirow{2}{*}{ Compound } & \multirow{2}{*}{$\begin{array}{c}\text { Concentration } \\
(\mathrm{mM})\end{array}$} & \multicolumn{3}{|c|}{ Uptake rate ( $\%$ of control) } \\
\hline & & & hMATE1 & hMATE2-K & rMATE $^{a}$ \\
\hline \multirow{11}{*}{$\mathrm{C}$} & None (control) & & $100 \pm 0.6$ & $100 \pm 1.2$ & $100 \pm 1.0$ \\
\hline & Amiloride & 0.2 & $5.2 \pm 1.2^{*}$ & $4.8 \pm 0.8^{*}$ & $7.9 \pm 0.8^{*}$ \\
\hline & Clonidine & 0.2 & $34.8 \pm 0.9^{*}$ & $49.1 \pm 3.2^{*}$ & $32.8 \pm 5.7^{*}$ \\
\hline & Diltiazem & 0.2 & $20.5 \pm 1.4^{*}$ & $68.2 \pm 2.0^{*}$ & $24.7 \pm 4.3^{*}$ \\
\hline & Diphenhydramine & 0.2 & $48.6 \pm 1.0^{*}$ & $77.8 \pm 1.8^{*}$ & $45.4 \pm 4.5^{*}$ \\
\hline & Imipramine & 0.2 & $23.5 \pm 2.7^{*}$ & $37.7 \pm 4.4^{*}$ & $30.2 \pm 4.0^{*}$ \\
\hline & MPP & 0.2 & $19.7 \pm 1.7^{*}$ & $17.8 \pm 1.4^{*}$ & $22.6 \pm 1.3^{*}$ \\
\hline & Norepinephrine & 0.2 & $93.0 \pm 0.6^{*}$ & $85.1 \pm 3.9^{*}$ & $106.2 \pm 4.9$ \\
\hline & Procainamide & 0.2 & $57.8 \pm 1.5^{*}$ & $47.2 \pm 1.9^{*}$ & $63.8 \pm 2.9^{*}$ \\
\hline & Quinidine & 0.2 & $11.0 \pm 2.0^{*}$ & $7.2 \pm 0.7^{*}$ & $15.0 \pm 2.2^{*}$ \\
\hline & Quinine & 0.2 & $16.8 \pm 1.1^{*}$ & $20.1 \pm 1.5^{*}$ & $15.8 \pm 2.6^{*}$ \\
\hline
\end{tabular}


Table 2 Continued...

\begin{tabular}{|c|c|c|c|c|c|}
\hline & Serotonin & 0.2 & $49.9 \pm 1.3^{*}$ & $50.9 \pm 1.0^{*}$ & $66.0 \pm 2.9^{*}$ \\
\hline & \multirow[t]{2}{*}{ TEA } & 0.2 & $71.8 \pm 1.9^{*}$ & $68.0 \pm 2.5^{*}$ & Not Determined \\
\hline & & 2 & $22.1 \pm 0.9^{*}$ & $21.5 \pm 1.8^{*}$ & $20.1 \pm 0.4^{*}$ \\
\hline & \multirow[t]{2}{*}{ Thiamine } & 0.2 & $10.1 \pm 1.0^{*}$ & $2.1 \pm 1.9^{*}$ & Not Determined \\
\hline & & 2 & $1.1 \pm 0.7^{*}$ & N.D. & $5.5 \pm 1.3^{*}$ \\
\hline & TPP & 0.2 & $18.8 \pm 0.6^{*}$ & $40.5 \pm 4.7^{*}$ & Not Determined \\
\hline & Verapamil & 0.2 & $28.1 \pm 1.1^{*}$ & $14.1 \pm 0.5^{*}$ & $23.9 \pm 3.2 *$ \\
\hline & DAPI & 0.1 & $4.3 \pm 0.8^{*}$ & $12.6 \pm 1.9^{*}$ & Not Determined \\
\hline & EB & 0.1 & $4.4 \pm 2.7^{*}$ & $0.4 \pm 0.4^{*}$ & Not Determined \\
\hline & PI & 0.1 & $17.1 \pm 2.4^{*}$ & $14.6 \pm 0.9^{*}$ & Not Determined \\
\hline & \multirow[t]{2}{*}{ Guanidine } & 0.2 & $95.2 \pm 2.9$ & $99.9 \pm 3.6$ & Not Determined \\
\hline & & 2 & $80.8 \pm 0.5^{*}$ & $104.8 \pm 2.5$ & $85.8 \pm 0.6^{*}$ \\
\hline & Histamine & 0.2 & $97.6 \pm 1.1$ & $101.9 \pm 3.0$ & $106.4 \pm 5.4$ \\
\hline & \multirow[t]{2}{*}{ Nicotinamide } & 0.2 & $103.1 \pm 2.0$ & $115.6 \pm 6.7^{*}$ & Not Determined \\
\hline & & 2 & $101.8 \pm 3.2$ & $106.9 \pm 2.5$ & $101.8 \pm 1.7$ \\
\hline & \multirow[t]{2}{*}{$\mathrm{NMN}$} & 0.2 & $106.4 \pm 0.9$ & $101.3 \pm 5.2$ & Not Determined \\
\hline & & 2 & $99.2 \pm 3.1$ & $91.3 \pm 2.1$ & $101.2 \pm 1.9$ \\
\hline $\mathrm{N}$ & Corticosterone & 0.2 & $38.2 \pm 2.1^{*}$ & $21.7 \pm 4.8^{*}$ & $19.3 \pm 1.9^{*}$ \\
\hline \multirow[t]{2}{*}{ A } & PAH & 0.2 & $106.0 \pm 3.8$ & $101.5 \pm 4.8$ & $102.0 \pm 3.8$ \\
\hline & Estrone-3-sulfate & 0.2 & $80.5 \pm 1.4^{*}$ & $96.7 \pm 4.9$ & $68.2 \pm 1.9^{*}$ \\
\hline
\end{tabular}

The uptake of $\left[{ }^{3} \mathrm{H}\right]$ cimetidine $(50 \mathrm{nM})$ was evaluated in the presence or absence of a test compound at $37^{\circ} \mathrm{C}$ and $\mathrm{pH} 7.4$ for $20 \mathrm{~s}$ after intracellular acidification by means of pretreatment with $\mathrm{NH}_{4} \mathrm{Cl}$ in HEK293 cells expressing hMATE1, those expressing hMATE2-K and mock cells. Data show the specific uptake rates by hMATE1 and hMATE2-K. ${ }^{a}$ Data for rMATE1 were cited from our previous report (4). The uptake rates for control were $1.74,0.85$ and $1.78 \mathrm{pmol} / \mathrm{min} / \mathrm{mg}$ protein, respectively, for hMATE1, hMATE2-K and rMATE1. Tested compounds are categorized into cationic $(\mathrm{C})$, anionic $(\mathrm{A})$ and neutral $(\mathrm{N})$ compounds. Data are represented as the means \pm S.E. $(n=3-5)$. *Significantly different from the value for control at $\mathrm{p}<0.05$; N.D., not detected. MPP, 1-methyl-4-phenylpyridinium; TEA, tetraethylammonium; TPP, tetraphenyl phosphonium; DAPI, 4',6-diamino-2-phenylindole; EB, ethidium bromide; PI, propidium iodide; $\mathrm{NMN}, \mathrm{N}^{1}$-methylnicotinamide; $\mathrm{PAH}, p$-aminohippurate.

Table 3. Parameters of the inhibitory effect of fluorescent cationic compounds on cimetidine uptake mediated by hMATEs stably expressed in HEK293 cells

\begin{tabular}{lcccc}
\hline Compound & Transporter & $\begin{array}{r}\mathrm{IC}_{50} \\
(\mu \mathrm{M})\end{array}$ & $n$ & $\begin{array}{c}v_{0} \\
(\mathrm{pmol} / \mathrm{min} / \mathrm{mg} \text { protein })\end{array}$ \\
\hline DAPI & hMATE1 & $6.9 \pm 0.7$ & $1.03 \pm 0.09$ & $1.85 \pm 0.03$ \\
& hMATE2-K & $15.7 \pm 1.9$ & $1.16 \pm 0.15$ & $0.92 \pm 0.05$ \\
EB & hMATE1 & $5.7 \pm 1.1$ & $1.08 \pm 0.19$ & $1.94 \pm 0.03$ \\
& hMATE2-K & $3.0 \pm 0.8$ & $1.02 \pm 0.20$ & $0.83 \pm 0.05$ \\
PI & hMATE1 & $15.3 \pm 1.8$ & $0.97 \pm 0.09$ & $2.21 \pm 0.03$ \\
& hMATE2-K & $29.1 \pm 3.7$ & $1.30 \pm 0.20$ & $0.74 \pm 0.04$ \\
\hline
\end{tabular}

Data are represented as the parameters \pm S.E. $(n=5$ or 6$)$ estimated by computer-fitting analyses of the data shown in Figure 4. $\mathrm{IC}_{50}$, half-inhibition concentration; $n$, Hill coefficient; $v_{0}$, uptake rate of $\left[{ }^{3} \mathrm{H}\right]$ cimetidine $(50 \mu \mathrm{M})$ in the absence of fluorescent test compounds. 
A

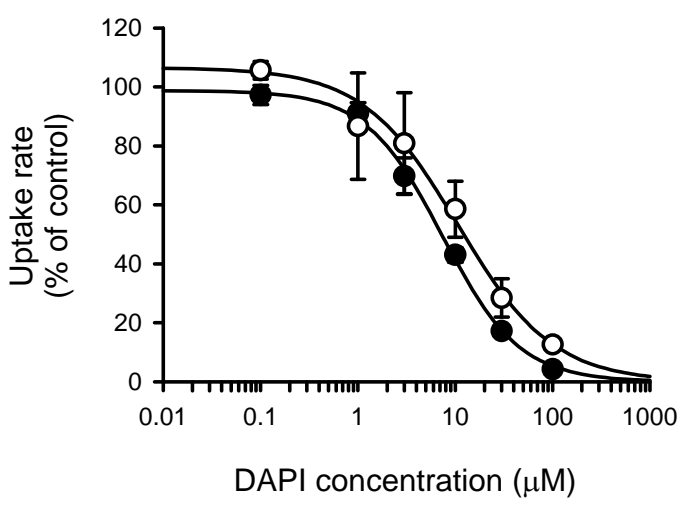

B

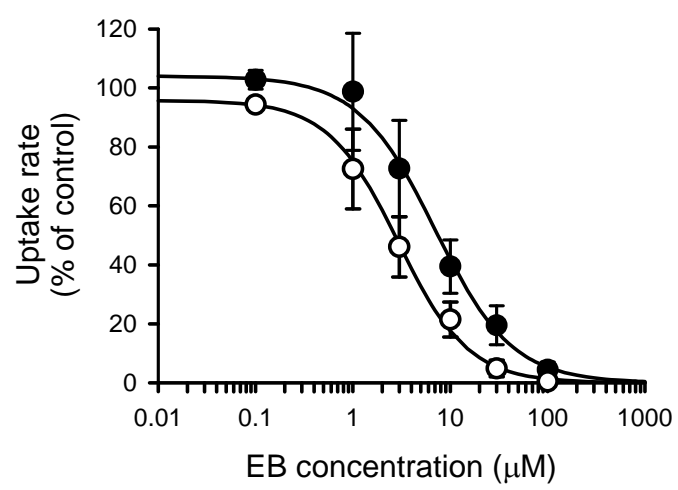

C

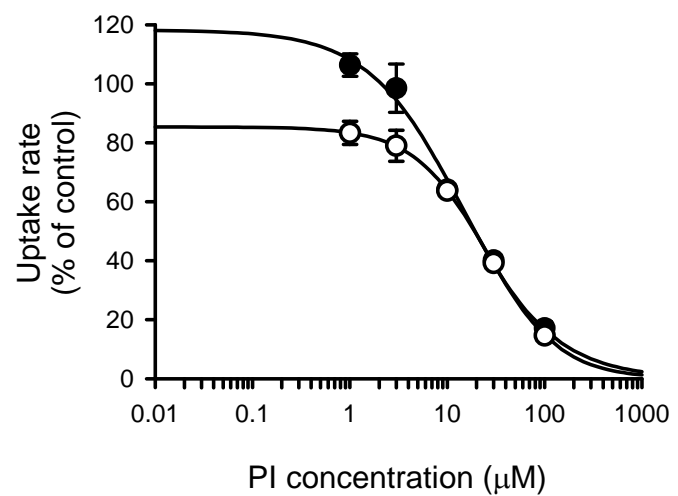

Figure 4. Inhibitory effect of fluorescent cationic compounds on cimetidine uptake mediated by hMATEs stably expressed in HEK293 cells. The uptake of $\left[{ }^{3} \mathrm{H}\right]$ cimetidine $(50 \mathrm{nM})$ was evaluated in the presence of varied concentrations of a test compound at $37^{\circ} \mathrm{C}$ and $\mathrm{pH} 7.4$ for $20 \mathrm{~s}$ after intracellular acidification by means of pretreatment with $\mathrm{NH}_{4} \mathrm{Cl}$ in $\mathrm{HEK} 293$ cells expressing hMATE1, those expressing hMATE2-K and mock cells. Data show the specific uptake rates by hMATE1 $(\bullet)$ and
hMATE2-K (०). Tested compounds are 4',6-diamino-2-phenylindole (DAPI), ethidium bromide (EB) and propidium iodide (PI), respectively, in panels A, B and $\mathrm{C}$. The values of observed $v_{0}$ for normalization of the uptake rate are 1.87 and $0.87 \mathrm{pmol} / \mathrm{min} / \mathrm{mg}$ protein, respectively, for hMATE1 and hMATE2-K. Solid lines represent the computer-fitted profiles and the parameters are shown in Table 3. Data are represented as the means \pm S.E. $(n=3-5)$.

\section{ACKNOWLEDGMENT}

This work was supported in part by a Grant-in-Aid for Young Scientists (B) from the Ministry of Education, Culture, Sports, Science and Technology, Japan (\#21790155).

\section{REFERENCES}

1. Otsuka, M., Matsumoto, T., Morimoto, R., Arioka, S., Omote, H., Moriyama, Y. A human transporter protein that mediates the final excretion step for toxic organic cations. Proc. Natl. Acad. Sci. U. S. A. 102: 17923-17928, 2005.

2. Terada, T., Yonezawa, A., Tanihara, Y., Kishimoto, K., Katsura, T., Ogawa, O., Inui, K. Identification and functional characterization of a new human kidney-specific $\mathrm{H}^{+}$organic cation antiporter, kidney-specific multidrug and toxin extrusion 2. J. Am. Soc. Nephrol. 17: 2127-2135, 2006.

3. Terada, T., Masuda, S., Asaka, J., Tsuda, M., Katsura, T., Inui, K. Molecular cloning, functional characterization and tissue distribution of rat $\mathrm{H}^{+}$/organic cation antiporter MATE1. Pharm. Res. 23: 1696-1701, 2006.

4. Ohta, K., Inoue, K., Hayashi, Y., Yuasa, H. Molecular identification and functional characterization of rat multidrug and toxin extrusion type transporter 1 as an organic cation $/ \mathrm{H}^{+}$antiporter in the kidney. Drug Metab. Dispos. 34: 1868-1874, 2006.

5. Hiasa, M., Matsumoto, T., Komatsu, T., Moriyama, Y. Wide variety of locations for rodent MATE1, a transporter protein that mediates the final excretion step for toxic organic cations. Am. J. Physiol. Cell Physiol. 291: C678-C686, 2006.

6. Matsushima, S., Maeda, K., Inoue, K., Ohta, K., Yuasa, H., Kondo, T., Nakayama, H., Horita, S., Kusuhara, H., Sugiyama, Y. The inhibition of human multidrug and toxin extrusion 1 is involved in the drug-drug interaction caused by cimetidine. Drug Metab. Dispos. 37: 555-559, 2009.

7. Tanihara, Y., Masuda, S., Sato, T., Katsura, T., Ogawa, O., Inui, K. Substrate specificity of MATE1 and MATE2-K, human multidrug and toxin extrusions $/ \mathrm{H}^{+}$-organic cation antiporters. Biochem. Pharmacol. 74: 359-371, 2007. 
8. Wigler, M., Silverstein, S., Lee, L. S., Pellicer, A., Cheng, Y., Axel, R. Transfer of purified herpes virus thymidine kinase gene to cultured mouse cells. Cell, 11: 223-232, 1977.

9. Boron, W. F., De Weer, P. Intracellular $\mathrm{pH}$ transients in squid giant axons caused by $\mathrm{CO}_{2}, \mathrm{NH}_{3}$, and metabolic inhibitors. J. Gen. Physiol. 67: 91-112, 1976.
10. Lowry, O. H., Rosebrough, N. J., Farr, A. L., Randall, R. J. Protein measurement with the Folin phenol reagent. J. Biol. Chem. 193: 265-275, 1951.

11. Yonezawa, A., Masuda, S., Yokoo, S., Katsura, T., Inui, K.: Cisplatin and oxaliplatin, but not carboplatin and nedaplatin, are substrates for human organic cation transporters (SLC22A1-3 and multidrug and toxin extrusion family). J. Pharmacol. Exp. Ther. 319: 879-886, 2006. 